\title{
Analysis of Alabama Airborne Gravity at Three Altitudes: Expected Accuracy and Spatial Resolution from a Future Tibetan Airborne Gravity Survey
}

\author{
Chi-Hsun Huang ${ }^{1}$, Cheinway Hwang ${ }^{1, *}$, Yu-Shen $\mathrm{Hsiao}^{2}$, Yan Ming Wang ${ }^{3}$, and Daniel R. Roman ${ }^{3}$ \\ ${ }^{I}$ Department of Civil Engineering, National Chiao Tung University, Hsinchu, Taiwan, ROC \\ ${ }^{2}$ Department of Soil and Water Conservation, National Chung Hsing University, Taichung, Taiwan, ROC \\ ${ }^{3}$ National Geodetic Survey, Silver Spring, Maryland, USA
}

Received 28 February 2012, accepted 30 October 2012

\begin{abstract}
In situ airborne gravity data at altitudes of $11,6.3$, and $1.7 \mathrm{~km}$ over a smooth area of Alabama are used to assess gravity accuracy and errors in upward and downward continuations. Analysis of the Alabama free-air anomaly gravity data at crossover points at the three altitudes suggests $1-2$ mgal accuracy for the dataset. Gravity data at each altitude are then expanded into local 3D Fourier series, to prepare for continuation. This Fourier representation results in continuation errors at few-mgal level in Alabama, even in the extreme case of downward continuation from $11 \mathrm{~km}$ to sea level. The result in Alabama inspires an airborne gravity survey over the rough, inaccessible terrain of Tibet. Similar investigations as in Alabama are made in Tibet using EGM08-derived airborne gravity data at flight altitudes of 10, 5, and $0 \mathrm{~km}$. Bouguer anomalies at the 10-km altitude preserve the major tectonic features of Tibet. Downward continuation errors increase with terrain roughness, but the survey can enhance local tectonic features. This study highlights the value of a future Tibetan airborne gravity survey and points out the expected gravity accuracy and spatial resolution from this survey.
\end{abstract}

Key words: Airborne gravity, Alabama, Downward continuation, Gravity anomaly, Tibet

Citation: Huang, C. H., C.Hwang, Y.S. Hsiao, Y.M.Wang, and D. R. Roman, 2013: Analysis of Alabama airborne gravity at three altitudes: Expected accuracy and spatial resolution from a future Tibetan airborne gravity survey. Terr. Atmos. Ocean. Sci., 24, 551-563, doi: 10.3319/TAO.2012.10.30.01(TibXS)

\section{INTRODUCTION}

Airborne gravimetry is an efficient and feasible tool to collect gravity data in inaccessible areas such as high mountains and coastal zones. Current reported accuracies are at the mgal level (Olesen et al. 2000; Hwang et al. 2007, 2012; Jordan et al. 2010). For an extreme terrain like Tibet, an airborne survey with a very high flight altitude (over $10 \mathrm{~km}$ ) is needed to avoid aviation hazards. At this altitude the gravity signal is significantly attenuated, making gravity recovery from the survey a challenging task (Verdun et al. 2003; Hwang et al. 2007). While there may be an airborne mission over Tibet at a high altitude in the future, no existing literature about achievable airborne gravity accuracy in such extreme conditions can be found.

In 2008 the National Geodetic Survey (NGS) of the US carried out 25 flights along the coastal region of Alabama, bordered by $27^{\circ} \leq$ latitude $\leq 31.5^{\circ}, 271.5^{\circ} \leq$ longitude $\leq$

\footnotetext{
* Corresponding author

E-mail: cheinway@mail.nctu.edu.tw
}

273.5 (GRAV-D Science Team 2011a, b; http://www.ngs. noaa.gov/GRAV-D/). Over this region airborne gravity data were collected in three campaigns at the altitudes of 1.7,6.3, and $11 \mathrm{~km}$. Because the campaigns flew the same geographically-located lines three times (once at each height), it is possible to continue gravity values from one altitude (starting altitude) to another (ending altitude), knowing that the continued data can be compared to the data collected at the other altitude. The continued gravity values at the ending altitude are then compared with the in situ measurements to assess the downward or upward continuation performance. The continuation from 11 to $1.7 \mathrm{~km}$ is of particular interest because this case is very likely to occur in Tibet. The result will provide an important reference for a future airborne gravity survey in Tibet.

Despite the fact that several papers have published results using land gravity measurements in Tibet, there is no publically released gravity in Tibet available to the authors of this paper. Examples of gravity applications in Tibet are 
large-scaled folding (Jin et al. 1994), lithospheric structure (Braitenberg et al. 2000), geoid modeling (Shen et al. 2011), and isostatic compensation (Tseng et al. 2009). Terrestrial gravity data used in these studies are mostly distributed in limited regions or along profiles that are accessible (roads or mountain tracks). Gravity fields from satellite missions such as GRACE (Shin et al. 2007) and GOCE (Pail et al. 2011) may provide uniform spatial coverage of Tibetan gravity, but the spatial resolutions are limited by the resolvable gravity field wavelengths (spherical harmonic degree 120 for GRACE and 240 for GOCE).

The problems of uneven spatial coverage and low spatial resolution of Tibetan gravity can be overcome by an airborne gravity mission in Tibet. Because of the high altitude of the Tibetan Plateau, for aviation safety the flight altitude of such a mission will be very likely to exceed $10 \mathrm{~km}$. At $10 \mathrm{~km}$ the theoretical resolvable gravity signal wavelength will be attenuated to about $5 \mathrm{~km}$ (Hwang et al. 2007), which is further degraded by data noise. The downward continuation of such high-altitude gravity data to sea level poses additional problems.

With this background, the objective of this paper is first to assess the accuracy of the Alabama airborne gravity in view of downward and upward continuations. We will then use the global gravity field model EGM08 (Pavlis et al. 2012) to generate simulated high-frequency gravity data in Tibet to repeat the assessment made in Alabama. Because the terrains and gravity signal strengths over Alabama and Tibet are substantially different, we expect different outcomes from these two regions. Lessons learned from this comparison will lead to better planning and understanding of a future Tibetan gravity survey. A more analogous study to one in Tibet could be attained from high-altitude data over the tallest mountain chain in North America (the Alaska Range), which would be possible once the GRAV-D data already collected there becomes publically available.

\section{ALABAMA AIRBORNE GRA VITY DATA AT THREE ALTITUDES}

\subsection{Data Description}

One of the important components of NOAA's "the Gravity for the Redefinition of the American Vertical Datum (GRAV-D)" project (GRAV-D Science Team 2011a, b; http://www.ngs.noaa.gov/GRAV-D/) is the airborne gravity survey over the whole United States and its territories. In 2007 the US National Geodetic Survey (NGS) acquired a new Turnkey Airborne Gravity System (TAGS) from the Micro-G/LaCoste company. NGS announced the first public release of airborne gravity data on September 5, 2011. The released data block contains airborne gravity lines collected during the Alabama 2008 survey flown from Montgomery, Alabama in January and February, 2008. The data block covers an area of $100 \times 400 \mathrm{~km}$ in the Gulf of Mexico and a small portion of the Alabama coastline. More information about the GRAV-D and TAGS can be found at http://www. ngs.noaa.gov/GRAV-D/. The aircraft flying the survey was an NOAA Cessna Citation II aircraft. The GPS positions were obtained from the Applanix PosPac software, doing a loosely-coupled GPS/IMU solution. Final horizontal and vertical position accuracy are estimated at 2.8 and $2.7 \mathrm{~cm}$, respectively (GRAV-D Science Team 2011b).

To see the TAGS gravimeter performance NGS carried out 25 flights in the coastal region over Alabama (see section 1). As stated before, the three flight altitudes were 1.7, 6.3 , and $11 \mathrm{~km}$. Figure 1 shows the survey line distributions. Such varying altitudes enable the continuation (or conversion) of the gravity signal from one (starting) altitude to another (ending) altitude for assessing continuation method performance. These datasets were collected at one-second intervals (one HZ). For the 1.7- and 6.3-km surveys, the line spacing is $10 \mathrm{~km}$ and the number of main lines in the northsouth direction is 10 (GRAV-D Science Team 2011b). For the $11-\mathrm{km}$ survey, the line spacing is $5 \mathrm{~km}$ and the number of the main lines is 20 . For all surveys, there are two westeast lines providing crossover differences for accuracy assessments. For clarity, although the raw data were provided by NGS. The results (from raw data to gravity anomaly) presented here were computed using methods used in Taiwan (Hwang et al. 2007). There may be differences between the results here and the NGS published version.

\subsection{Gravity Accuracy Assessment by Crossover Differ- ence and Continuation}

Figure 1 shows the distribution of gravity anomaly crossover differences and Table 1 shows the statistics of the differences from the three surveys. The root-mean-squared (RMS) differences from the crossover adjusted gravity values are also included in Table 1. The crossover adjustment based on a bias-only model, reduces the crossover differences by up to $1.347 \mathrm{mgal}$ ( $1.7 \mathrm{~km}$ altitude). The crossover differences do not depend on altitude and the case at $6.3 \mathrm{~km}$ has the largest difference. Table 1 suggests that the overall gravity accuracies (in terms of RMS crossover differences) of the Alabama data are at the $1-2$ mgal level for all altitudes. Gravity anomalies at the three altitudes are shown in Fig. 2. The patterns of the major gravity signatures from the three surveys are quite similar. As expected, the gravity field detail and its local high and low increase with decreasing altitude, especially in the Gulf of Mexico. Because of a smaller line spacing $(5 \mathrm{~km})$ in the $11-\mathrm{km}$ survey, the gravity contours appear to vary faster than the other cases, but this rapid variation does not reflect the high-frequency gravity signal.

In a typical airborne survey the flight altitude is maintained at the same value throughout the survey. However, the flight altitude may be adjusted during a survey for the 
(a)

(b)
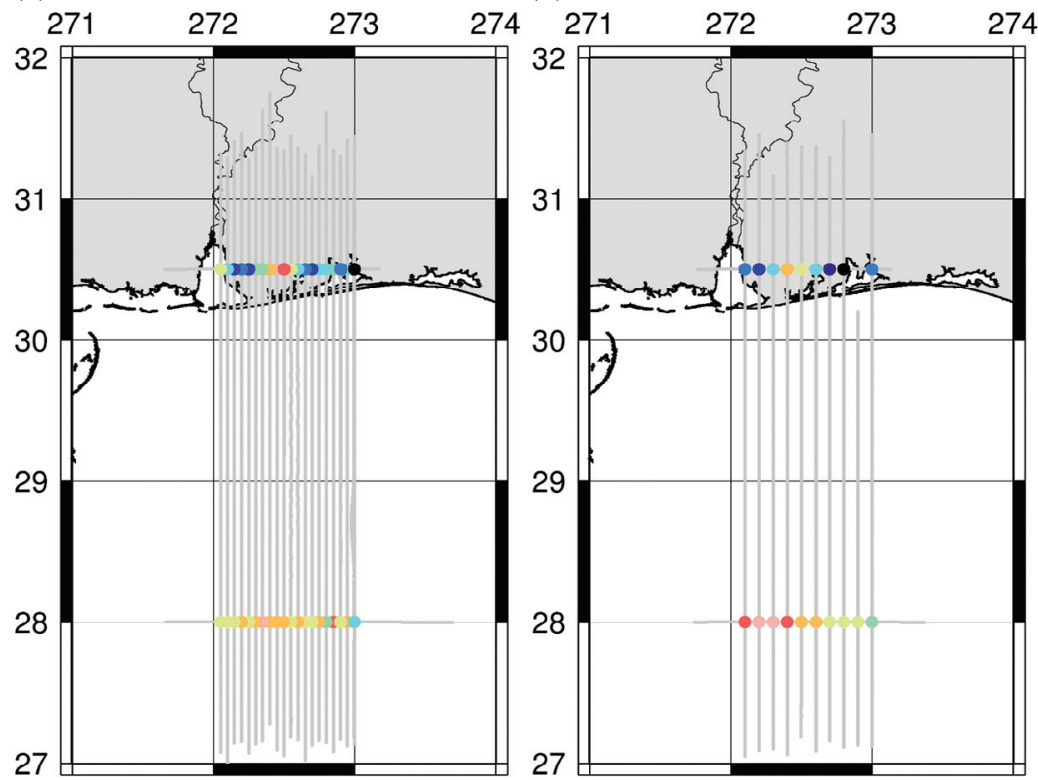

(c)

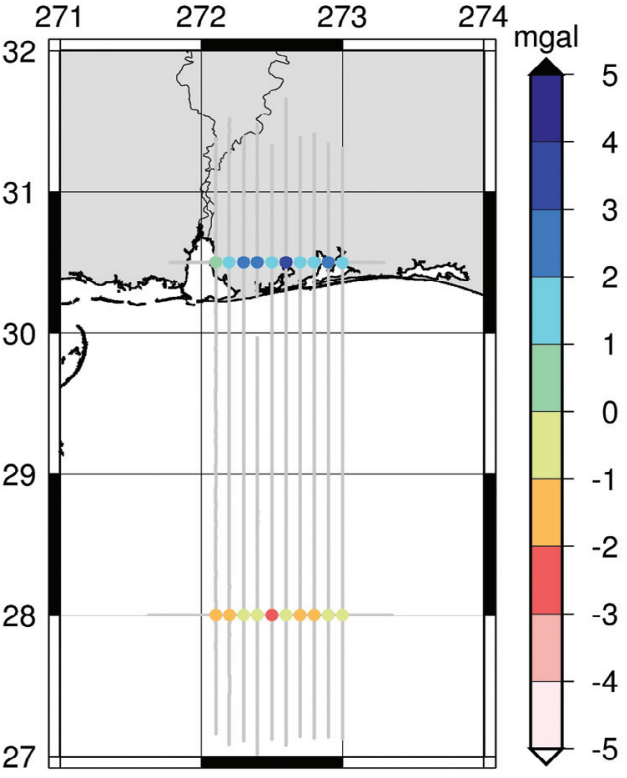

Fig. 1. Crossover differences of gravity anomaly at 3 different altitudes (Alabama): (a) $11 \mathrm{~km}$, (b) $6.3 \mathrm{~km}$, and (c) $1.7 \mathrm{~km}$.

Table 1. Statistics of crossover differences of gravity anomaly in Alabama (in mgal) at three altitudes (in km).

\begin{tabular}{ccccccc}
\hline Altitude & Max & Min & Mean & Std. dev. & RMS & RMS $^{\text {a }}$ \\
\hline 1.7 & 3.25 & -2.64 & 0.29 & 1.65 & 1.67 & 0.31 \\
6.3 & 7.61 & -1.42 & 1.17 & 2.05 & 2.29 & 1.21 \\
11 & 4.15 & -2.32 & 1.02 & 1.63 & 1.91 & 0.73 \\
\hline
\end{tabular}

aith crossover adjustment.

(a)

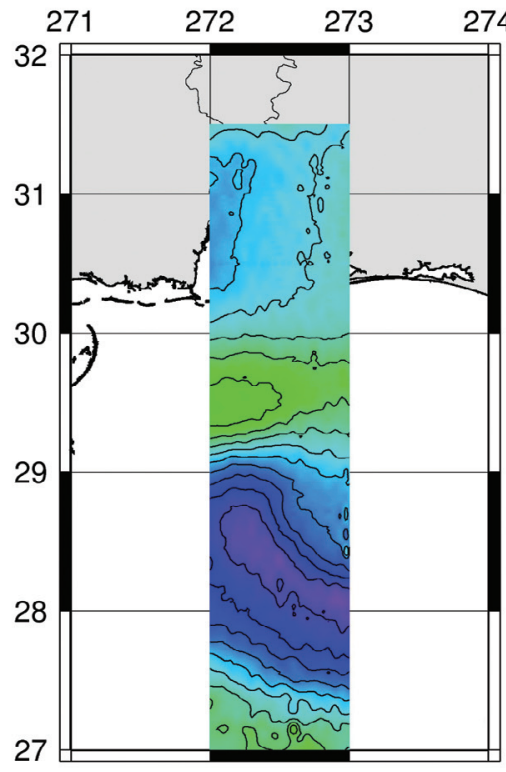

(b)

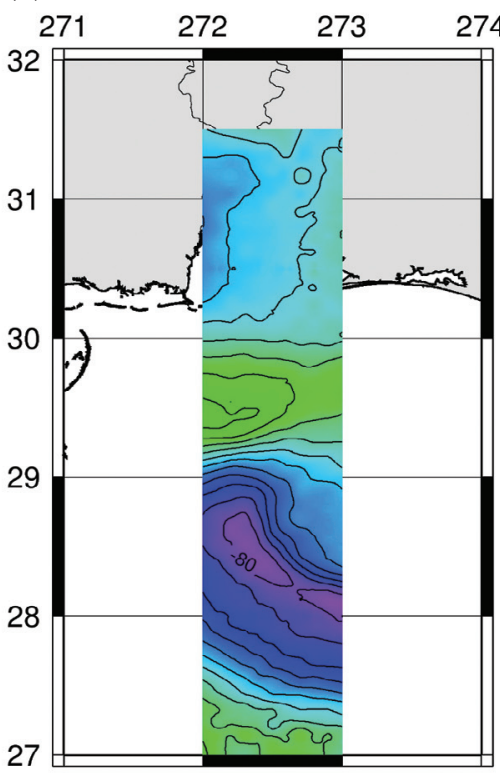

(c)

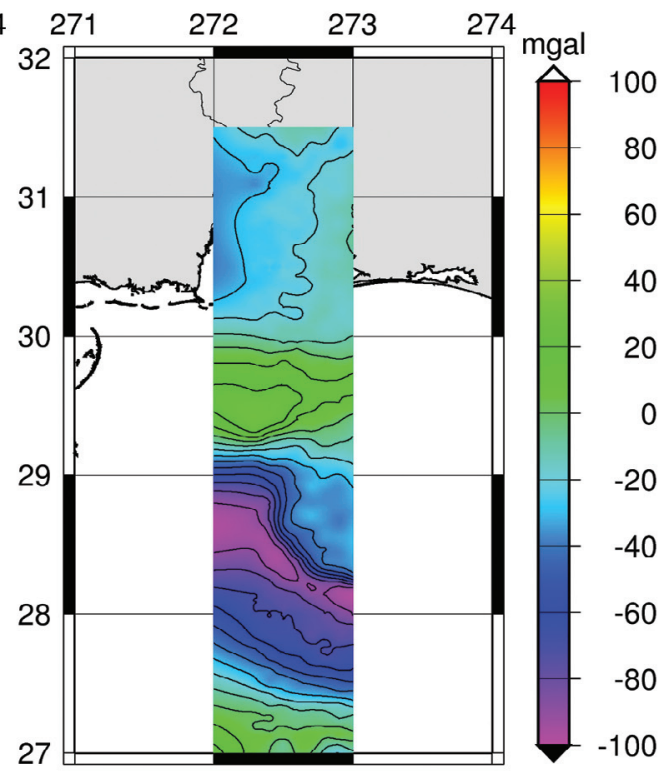

Fig. 2. Airborne gravity anomalies at 3 different altitudes (Alabama): (a) $11 \mathrm{~km}$, (b) $6.3 \mathrm{~km}$, and (c) $1.7 \mathrm{~km}$. 
sake of flight safety or visualization. In some cases the altitude adjustment can be over hundreds of meters, leading to changes in gravity caused by the gravity gradient. If the varying data collection altitudes are ignored height-induced errors will be introduced into the airborne data processing result. Therefore, there will be errors in the continuation products if an average altitude is used. For clarity, the altitudes of $1.7,6.3$, and $11 \mathrm{~km}$, which are the mean altitudes for the three surveys, are used only to represent the three flight campaigns but not used in any computation procedure.

The continuations in this paper are based on the remove-restore approach. Specifically, at a given starting flight altitude, the residual gravity is obtained by subtracting the long wavelength part from the measured gravity values (these values refer to the flight altitude), and then continued to an ending altitude. The long wavelength part is based on an EGM08 expansion from degree 2 to 720 . Finally, the long wavelength part at the ending altitude is restored and the resulting gravity values are computed. To cope with the varying data collection altitudes we model the gravity field using the 3D Fourier series of Wang et al. (2008), which includes the flight altitude effect. Specifically, a residual airborne gravity anomaly at position $(x, y, h)$ is modeled as

$$
\begin{aligned}
\delta g(x, y, h)= & \sum_{n=0}^{N} \sum_{m=0}^{M} \lambda_{n m}\left(a_{n m} \cos \xi_{n} x \cos \eta_{m} y\right. \\
& +b_{n m} \cos \xi_{n} x \sin \eta_{m} y+c_{n m} \sin \xi_{n} x \cos \eta_{m} y \\
& \left.+d_{n m} \sin \xi_{n} x \sin \eta_{m} y\right) \omega_{n m} e^{-\omega_{n m} h}
\end{aligned}
$$

where

$\xi_{n}=2 n \pi / 2 L_{x} ; \quad \eta_{m}=2 m \pi / 2 L_{y}$

$\omega_{n m}=\sqrt{\xi_{n}^{2}+\eta_{m}^{2}}$

$\lambda_{n m}= \begin{cases}0.25 & (m=0, n=0) \\ 0.5 & (m>0, n=0 ; m=0, n>0) \\ 1 & (m>0, n>0)\end{cases}$

with $x$ and $y$ : the local coordinate ( $x$ to the east and $y$ to the north). The origin of $x$ and $y$ is at the center of a given area; $M$ and $N$ : the maximum numbers of $m$ and $n$, representing the Nyquist frequencies (Buttkus 2000); $h$ : the flight altitude; $a_{n m}, b_{n m}, c_{n m}$, and $d_{n m}$ : coefficients of the Fourier expansion; $L_{x}$ and $L_{y}$ : the half width (west-east dimension) and half length (south-north dimension) of the area.

With altitude $(h)$ in the model, Eq. (1) can be used for downward and upward continuations as follows. The airborne gravity observations at any altitudes can be used to estimate the Fourier coefficients in Eq. (1). In the matrix representation the observation equations read
$\mathbf{V}=\left[\begin{array}{c}v_{1} \\ \ldots \\ v_{k}\end{array}\right]=\left[\begin{array}{c}\mathbf{a}_{1}\left(x_{1}, y_{1}, h_{1}\right) \\ \ldots \\ \mathbf{a}_{k}\left(x_{k}, y_{k}, h k\right)\end{array}\right]\left[\begin{array}{c}a_{00} \\ \ldots \\ d_{N M}\end{array}\right]-\left[\begin{array}{c}\delta g_{1} \\ \ldots \\ \delta g_{k}\end{array}\right]=\mathbf{A X}-\mathbf{L}$

where $\mathbf{A}$ is the design matrix, vectors $\mathbf{X}, \mathbf{L}$ and $\mathbf{V}$ contain respectively parameters (coefficients), observations and residuals, and $k$ is the number of observations. The least-squares solution of Eq. (5) with a priori covariance matrix of the coefficients $\mathbf{C}_{\mathbf{X}}$ is (Liebelt 1967; Koch 1999)

$\hat{\mathbf{X}}=\left(\mathbf{C}_{\mathbf{X}}^{-1}+\mathbf{A}^{\mathrm{T}} \mathbf{C}_{\mathrm{v}}^{-1} \mathbf{A}\right)^{-1} \mathbf{A}^{\mathrm{T}} \mathbf{C}_{\mathrm{v}}^{-1} \mathbf{L}$

where $\mathbf{C}_{\mathbf{v}}$ is the error covariance matrix of the observations. Because of the difficulties in modeling the airborne gravity correlation values, in this paper we ignore the correlations and therefore assume that $\mathbf{C}_{\mathbf{v}}$ is a diagonal matrix. Understanding the gravity correlations and how they can improve the estimation of $\hat{\mathbf{X}}$ in Eq. (6) is a subject of future study. Based on Table 1, an error variance of $4 \mathrm{mgal}^{2}$ is assigned to the diagonal elements of $\mathbf{C}_{\mathrm{v}} \cdot \mathbf{C}_{\mathbf{x}}^{-1}$. will stabilize the leastsquares solution of Eq. (5).

For a $10-\mathrm{km}$ line spacing (about 5 arc minutes), the values of $N$ and $M$ corresponding to the Nyquist frequencies of the Fourier series are 25 and 55, respectively. These numbers are doubled in the case of $11-\mathrm{km}$ survey because the flight lines are twice as dense. We assume that $\mathbf{C}_{\mathrm{x}}^{-1}$ is a diagonal matrix, with the diagonal elements $f$ computed as

$f=1 /\left[\alpha\left(n^{2}+m^{2}\right)+1\right]$

where $\alpha$ is an empirical regularization parameter. The degree of smoothness of the solution increases with the value of $\alpha$. We find that, with $\alpha=15$, the differences between continued and in situ gravity values (see below) are the smallest. In this paper, we use this value for all solutions of the Fourier coefficients.

The model in Eq. (1) is assessed using a series of experiments as follows. We carried out the following cases of upward continuation and accuracy assessment first:

Case I: gravity measurements at 1.7 and $6.3 \mathrm{~km}$ are upward continued to $11 \mathrm{~km}$, and the two resulting values are compared at $11 \mathrm{~km}$ (low and medium altitudes to high altitude); Case II: gravity measurements at $1.7 \mathrm{~km}$ are upward continued to $11 \mathrm{~km}$, and the resulting values are compared with the measurements at $11 \mathrm{~km}$ (low to high altitude);

Case III: gravity measurements at $6.3 \mathrm{~km}$ are upward continued to $11 \mathrm{~km}$, and the resulting values are compared with the measurements at $11 \mathrm{~km}$ (medium to high altitude).

These three cases are designed to see the impact of continuation distance (defined as the difference between two altitudes) on the accuracy of continued gravity, in con- 
nection to the simulation study of a future Tibetan airborne gravity mission (section 3 ). Table 2 shows the statistics for the differences in the three cases. Despite the usual errors in airborne gravimetry due to varying aircraft response to winds/turbulence and errors in GPS positioning and GPSderived accelerations for the three gravity surveys, the standard deviations of the differences in Table 2 are quite close to the standard deviations of crossover differences (Table 1). The mean differences ranged from 1.24 to $3.66 \mathrm{mgal}$, which are significant compared to the magnitude of gravity here. These three comparisons highlight the fact that upward continuation of airborne gravity data will introduce errors in the data, particularly with respect to the mean values of the continued data.

The next assessment is on the downward continuation performance of the 3D Fourier series. Gravity measurements were downward continued from one altitude to another in the following three cases:

Table 2. Statistics of the differences (in mgal) from comparisons in Cases I, II, and III.

\begin{tabular}{crrcc}
\hline Case & Max & Min & Mean & Std. dev. \\
\hline I & 9.59 & -1.79 & -2.41 & 0.96 \\
II & 11.99 & -0.67 & 3.66 & 1.08 \\
III & 3.48 & -0.38 & -1.24 & 0.52 \\
\hline
\end{tabular}

Case A: downward continue from 11 to $6.3 \mathrm{~km}$ (high to medium altitude);

Case B: downward continue from 6.3 to $1.7 \mathrm{~km}$ (medium to low altitude);

Case C: downward continue from 11 to $1.7 \mathrm{~km}$ (high to low altitude).

The resulting gravity values (Fig. 3) were then compared with the in situ measurements at the altitudes of $6.3 \mathrm{~km}$ (Case 1) and $1.7 \mathrm{~km}$ (Cases B and C). Table 3 shows the statistics of the differences in the three cases. Figure 4 shows the differences between downward continued and true gravity values in Alabama from three cases. Comparison between Fig. 2 and Fig. 3 shows that most gravity signatures are preserved in the downward continued fields. Based on Table 3, Case A, and Case C are for high $(11 \mathrm{~km})$ to medium $(6.3 \mathrm{~km})$ and for high $(11 \mathrm{~km})$ to low $(1.7 \mathrm{~km})$. The difference between the these downward continuation distances $5 \mathrm{~km}$, and the standard deviation (Table 3) increases from
Table 3. Statistics of differences (in mgal) between downward-continued and airborne gravity values from Cases A, B, and C (Alabama).

\begin{tabular}{crrcc}
\hline Case & Max & Min & Mean & Std. dev. \\
\hline A & 9.98 & -7.72 & -0.91 & 1.48 \\
B & 9.41 & -7.19 & -1.27 & 1.90 \\
C & 13.85 & -11.66 & -2.31 & 3.09 \\
\hline
\end{tabular}

(a)

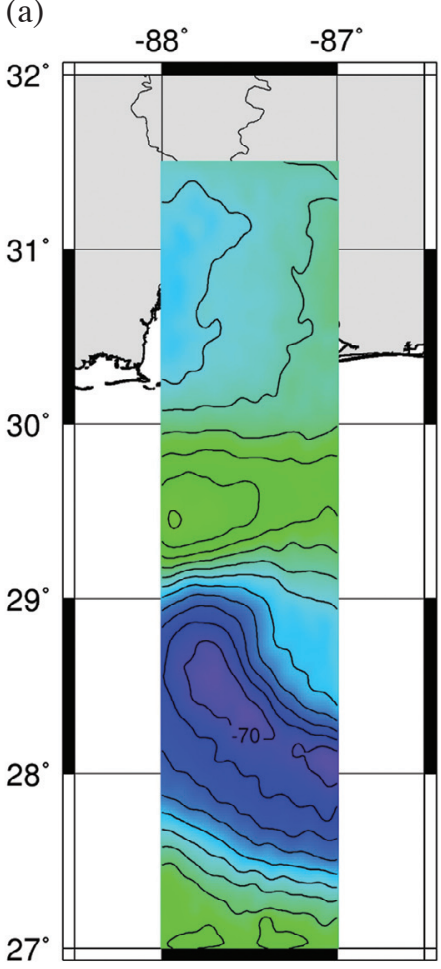

(b)

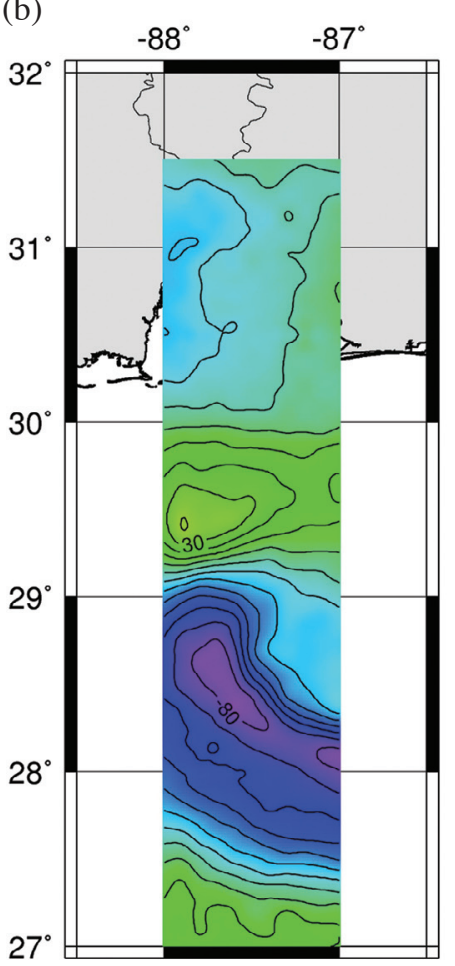

(c)

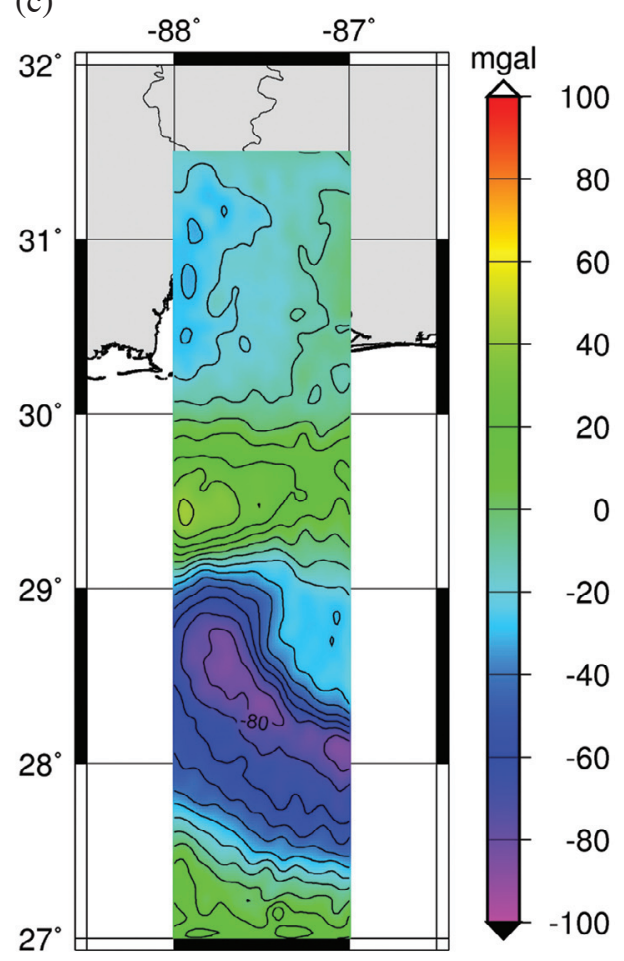

Fig. 3. Downward continuation results in Alabama from (a) Case A, (b) Case B, and (c) Case C. 
(a)

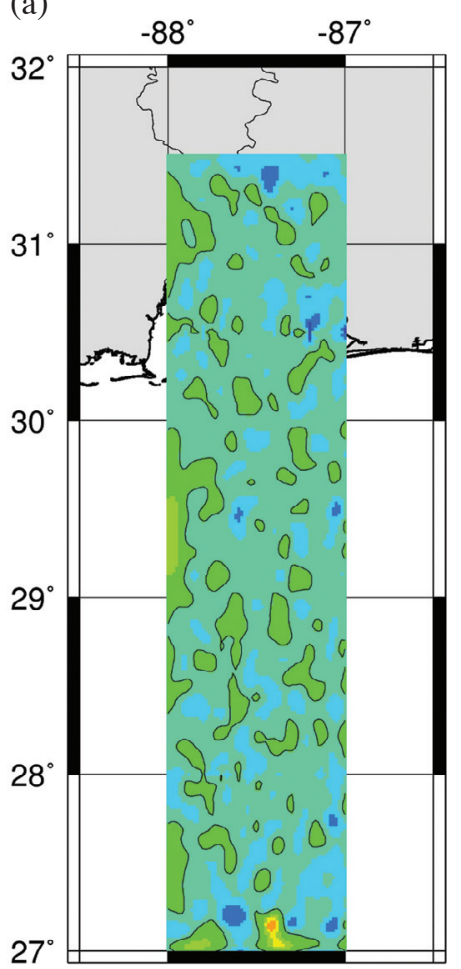

(b)

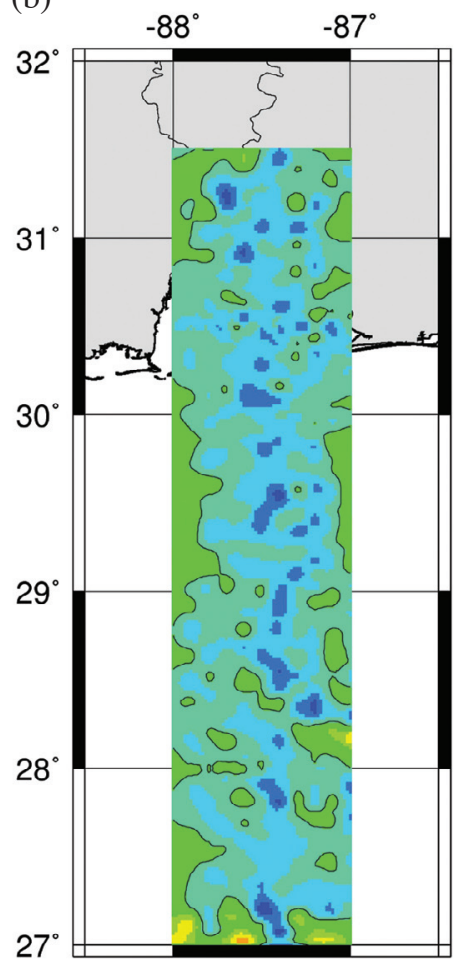

(c)

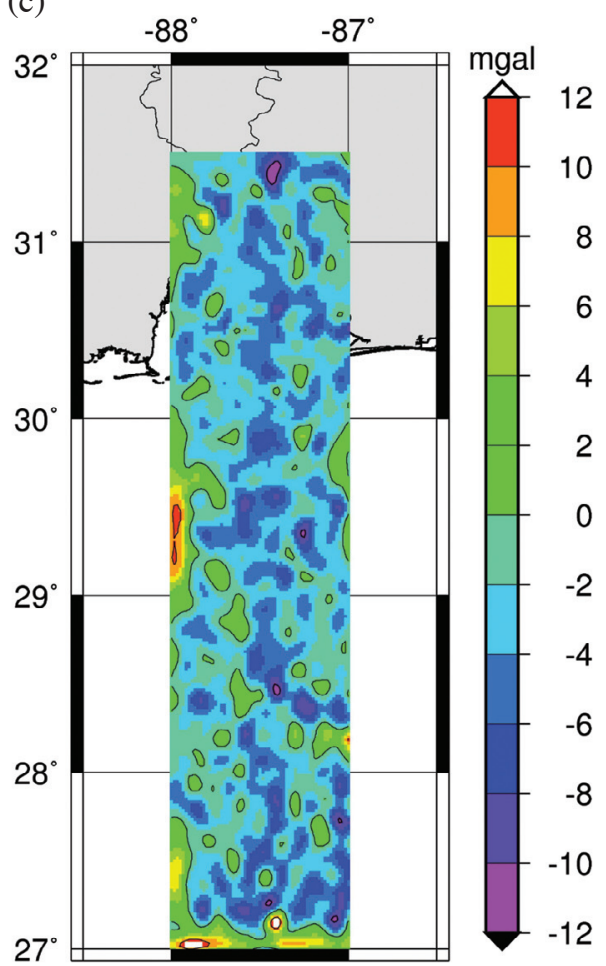

Fig. 4. Differences between downward continued and true gravity values in Alabama from (a) Case A, (b) Case B, and (c) Case C.

$1.48 \mathrm{mgal}$ (Case A) to $3.09 \mathrm{mgal}$ (Case C). This increase in standard deviation $(3.09-1.48=1.61 \mathrm{mgal})$ is marginal compared to the standard error of 1 - 2 mgal in the Alabama airborne gravity surveys. The noises are slightly amplified due to the increase of downward continuation length, but the amplification is marginal compared to the airborne gravity accuracy. The extreme differences of -11.66 and $13.85 \mathrm{mgal}$ in Table 3 can be due to outliers in the raw data, and it is recommended that data editing be made before using the Alabama airborne gravity. The gravity signatures and resolutions in Cases B and C are similar and the gravity field in Case A is rather smooth compared to those in Cases B and C. From the above experiments of upward and downward continuations, it is evident that the three gravity datasets at different altitudes are highly consistent. Over a coastal region such as Alabama, an airborne gravity survey at a high altitude is feasible and will deliver a promising result.

For applications such as geoid modeling and orthometric correction, it is necessary to downward continue gravity values from a given flight altitude to sea level. In this paper we also downward continued the gravity values at the three flight altitudes to sea level. The three cases of downward continuation are $11 \mathrm{~km}$ to 0 (Case D), $6.3 \mathrm{~km}$ to 0 (Case E) and $1.7 \mathrm{~km}$ to 0 (Case F), respectively. We then compared the results with the gravity values computed from EGM08 up to degree 2160 , corresponding to a $5^{\prime} \times 5^{\prime}$ spatial reso- lution. According to Pavlis et al. (2012), EGM08 includes all surface gravity data around the USA. Therefore, gravity anomalies from the expansion to degree 2160 are considered as ground measurements at the $5^{\prime} \times 5^{\prime}$ resolution level. Table 4 shows the statistics of the differences in Cases D, $\mathrm{E}$, and F. Even in the most challenging scenario of Case D $(11 \mathrm{~km}$ to 0$)$, the standard deviation of the differences is below $4 \mathrm{mgal}$. Note that the topography in this flight region is rather smooth. For such a mountainous terrain as Tibet, we may not achieve the same gravity accuracy as in Alabama.

\section{ANALYSIS OF SIMULATED AIRBORNE GRAV- ITY DATA OVER TIBET}

\subsection{Simulated Gravity Data over Tibet}

The Alabama gravity analysis suggests that an airborne gravity survey at a high altitude $(>10 \mathrm{~km})$ can deliver mgal-level gravity accuracy at sea level. Because any fu-

Table 4. Statistics of differences (in mgal) between downward-continued and EGM08 gravity values from Cases D, E, and F (Alabama).

\begin{tabular}{ccccc}
\hline Case & Max & Min & Mean & Std. dev. \\
\hline D & 16.74 & -15.37 & -1.52 & 3.79 \\
E & 13.46 & -14.79 & -0.42 & 3.16 \\
F & 12.25 & -15.19 & -0.91 & 2.56 \\
\hline
\end{tabular}


ture airborne gravity survey in Tibet will be flown at a high altitude, here we will see whether this accuracy level can be achieved in Tibet based on simulated gravity data. For the simulation, we generated gravity anomalies using the spherical harmonic expansion as

$$
\begin{aligned}
\Delta g(\varphi, \lambda, h)= & \frac{G M}{R} \sum_{n=2}^{n_{m x}}\left(\frac{R}{R+h}\right)^{n+1} \\
& \cdot \sum_{m=0}^{n}\left(\bar{C}_{n m} \cos m \lambda+\bar{S}_{n m} \sin m \lambda\right) \bar{P}_{n m} \sin \phi
\end{aligned}
$$

where $\varphi, \lambda$ are geocentric latitude and longitude, $\bar{C}_{n m}$ and $\bar{S}_{n m}$ are the fully normalized geopotential coefficients (EGM08 in this paper), $\bar{P}_{n m}$ is the fully normalized associated Legendre functions, $R$ is the semi-major axis of the reference ellipsoid and $h$ is the flight altitude, and $n_{\max }$ is the maximum degree of expansion. With $h$ changed from one value to another, the spherical harmonic expansion enables downward and upward continuations. However, with a dense gravity dataset as that given in Alabama, a representation by the spherical harmonic expansion will need a large $n_{\max }$ value, making the continuation computation less efficient. Instead, a local function such as the 3D Fourier series in Eq. (1) is computationally more efficient and stable.

Based on Eq. (8), we generated 3 gravity datasets at altitudes of 10,5 , and $0 \mathrm{~km}$, at an along-track spacing of $5 \mathrm{~km}$ from expansions to degree 2160 . We did not add data noise to the simulated gravity values because the added noise will be reduced by the filtering in Eq. (6). This filtering effect is seen in the Alabama test results (Tables 3 and 4). However, it is stressed that, if the future in situ airborne gravity data in Tibet contains noise much larger than 2 mgal, or even large systematic errors, the conclusion made in Tables 3 and 4 will not be valid. The three different altitudes are similar (1.7 km or less) to the Alabama altitudes. The distributions of the three sets of simulated survey lines are shown in Fig. 5. The gravity values from EGM08 in Tibet are considered as "true" ones, as those collected in the Alabama airborne surveys. Like Alabama, we can assess the downward continuation or upward continuation performance using Eq. (1) by comparing the continued gravity values with the "true" ones. The elevations of the flight area $\left(27^{\circ} \leq \varphi\right.$ $\leq 31.5^{\circ}, 91.5^{\circ} \leq \lambda \leq 93.5^{\circ}$ ) based on SRTM range from 77 to $7003 \mathrm{~m}$, with a standard deviation of $1264 \mathrm{~m}$. For comparison, the elevations in the flight area of Alabama (Fig. 1) range from -3057 to $167 \mathrm{~m}$ and the standard deviation is $1229 \mathrm{~m}$ with a mean value of $-1030 \mathrm{~m}$. On land, the elevations along the survey lines in Alabama range from 0 to $167 \mathrm{~m}$, with a standard deviation of only $33 \mathrm{~m}$. Thus the land area of the flight can be considered as a plain. Because of different terrains in the two areas, we expect different outcomes of downward and upward continuations of gravity anomalies.
Because Eq. (1) was proven to be effective in Alabama and downward continuation is more challenging than upward continuation, in Tibet we only experiment with downward continuation. Following the procedure in Alabama, the long wavelength part is represented by an expansion from degree 2 to 720 . At a given starting flight altitude, the residual gravity is obtained by subtracting the long wavelength part from the total part (to degree 2160), and then downward continued to a lower (ending) altitude. Finally, the long wavelength part at the ending altitude is restored and the resulting gravity values are compared with the "true" gravity values. Because the simulated gravity datasets are produced by the same (errorless) geopotential coefficients of EGM08, the crossover differences will be automatically zero.

\subsection{Analysis of Tibetan Simulated Airborne Gravity Data}

We have experimented with 3 cases of assessment of downward continuation accuracy in Tibet as follows:

Case G: downward continue from 10 to $5 \mathrm{~km}$;

Case $\mathrm{H}$ : downward continue from $5 \mathrm{~km}$ to sea level $(0 \mathrm{~km})$; Case I: downward continue from $10 \mathrm{~km}$ to sea level.

Figure 6 shows the gravity values from the downward continuations. Table 5 shows the statistics for the differences between the downward-continued gravity values and the EGM08-generated "true" ones from Cases G, H and I.

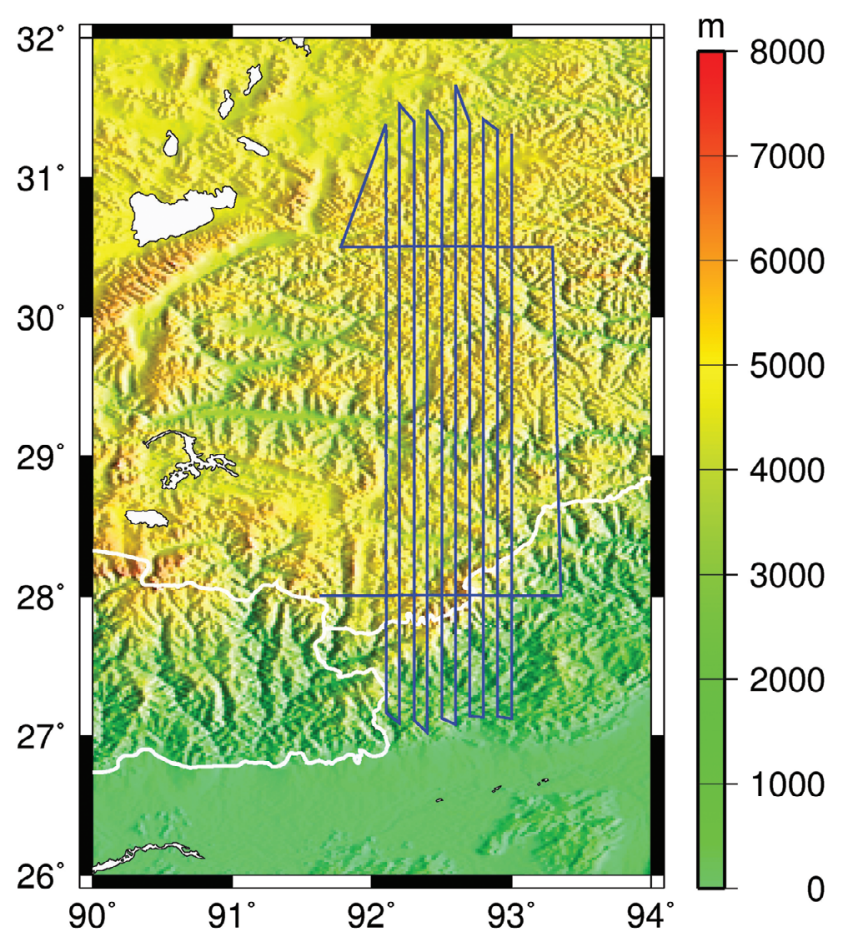

Fig. 5. The distribution of simulated flight lines and the terrain from SRTM over Tibet. 
(a)

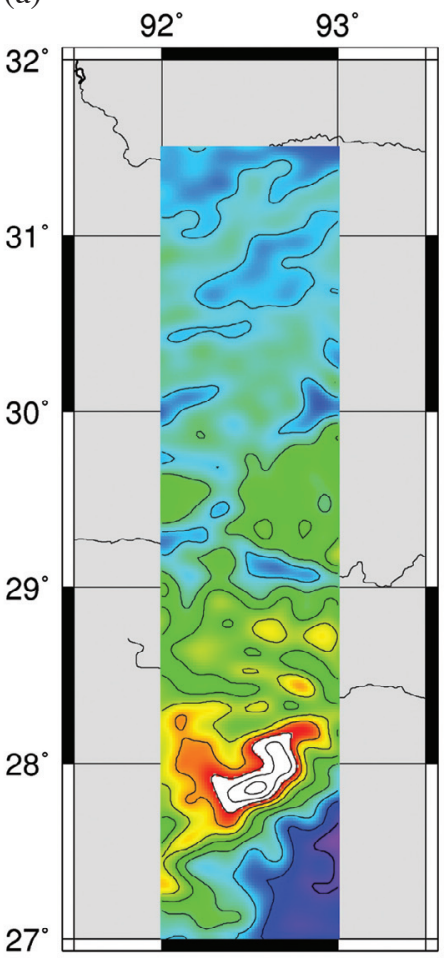

(b)

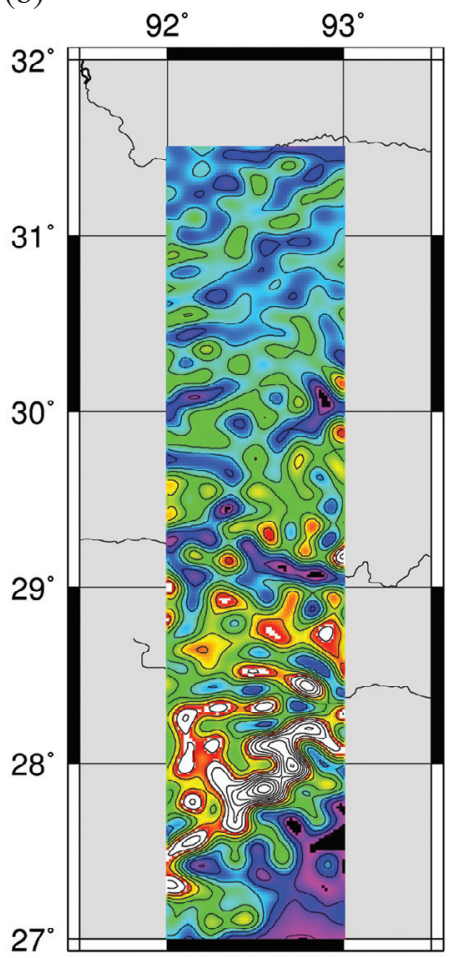

(c)

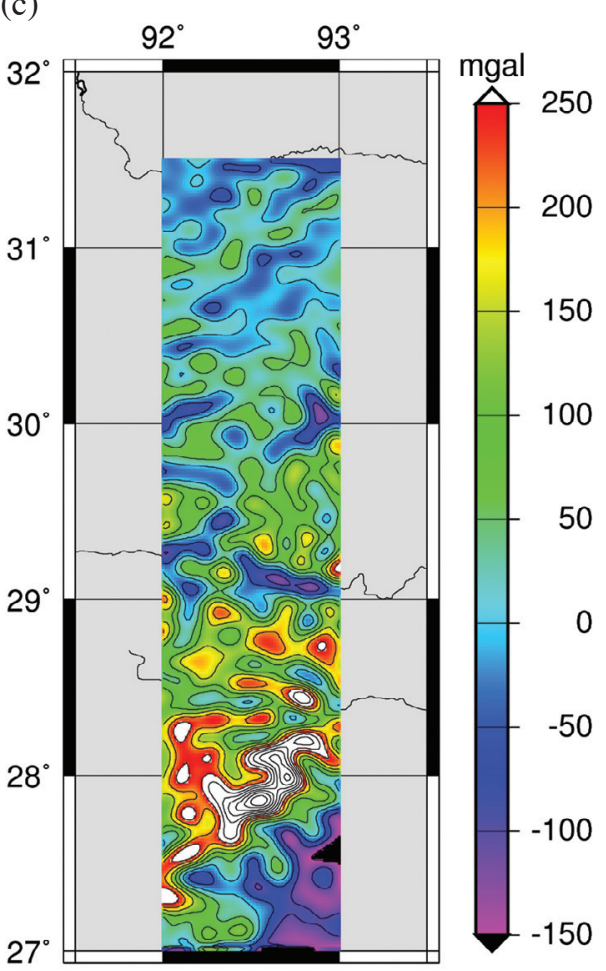

Fig. 6. Gravity anomalies from downward continuation in Tibet: (a) Case G, (b) Case H, and (c) Case I.

Table 5. Statistics of differences (in mgal) between downward-continued and EGM08 gravity values from Cases G, H, and I (Tibet).

\begin{tabular}{ccccc}
\hline Case & Max & Min & Mean & Std. dev. \\
\hline G & 16.33 & -18.67 & -0.01 & 3.47 \\
H & 50.95 & -51.52 & -0.01 & 11.41 \\
I & 155.85 & -110.47 & -0.02 & 22.62 \\
\hline
\end{tabular}

Figure 7 compares the differences in downward continued and "true" gravity values in Tibet. The relatively small gravity differences (at few mgal) from Cases A and G suggest that, if the ending altitude in Tibet is near the average elevation, the downward continuation error is significantly smaller, compared to the case of downward continuation below the average elevation. Compared to the three Alabama cases (Table 4), the standard deviations of the differences in Tibet (Table 5) are larger, with the difference increasing from Cases $\mathrm{G}$ to I. The maximum differences in Tibet exceed 100 mgal (Case I), compared to about 10 mgal in Alabama (Table 3). In Alabama, downward continuation with a long continuation distance (Case C) does not deteriorate the gravity data, but such deterioration is evident in Tibet (Case I). This is also true when comparing Case B and Case H. Although Cases B and H are both cases of downward continuations from a medium to a low altitude, the results are quite different. Figure 8 shows the differences between the downward-continued gravity values (Case I) and the true values, and the correlation with the topography. The areas with large differences are within the red and yellow circular zones marked in Fig. 8, which contain two deep valleys. In general, the gravity difference increases with the terrain gradient. From the case studies in Alabama and Tibet we conclude that the downward continuation error increases with terrain roughness and is not correlated with the elevation magnitude.

\section{RESOLUTION OF AIRBORNE BOUGUER GRAV- ITY ANOMALIES IN TIBET}

The Bouguer anomaly (BA) is commonly used in studying crustal structure and density contrast. The BA is obtained by removing the topography gravity effect above sea level from the free-air gravity anomaly (called Bouguer reduction). To see the airborne gravity limitation in picking up a high frequency gravity signature in Tibet, we also computed the BA at an altitude of $10 \mathrm{~km}$ and then downward continued the gravity values to sea level. The topographic effect for the Bouguer reduction was computed using the Gauss quadrature method (Hwang et al. 2007). The density model used here is $\rho_{0}=2.67 \mathrm{~g} \mathrm{~cm}^{-3}$. Figure 9 shows the BA at $10 \mathrm{~km}$ and at sea level (from downward continuation). As expected, the downward continuation enhanced the local gravity signature. However, the enhancement extent is not 
(a)

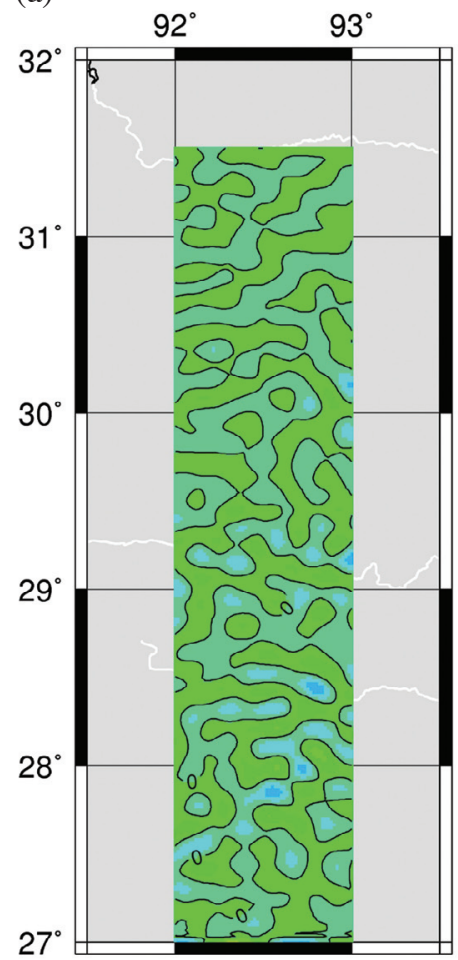

(b)

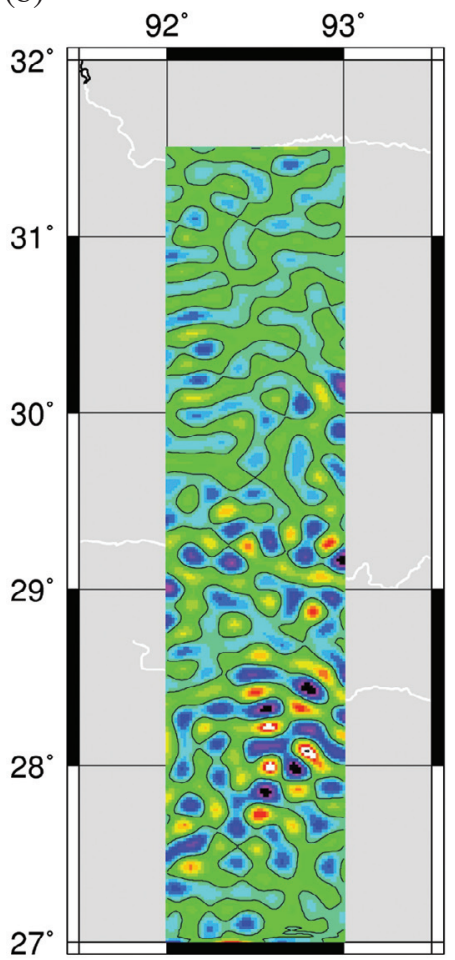

(c)

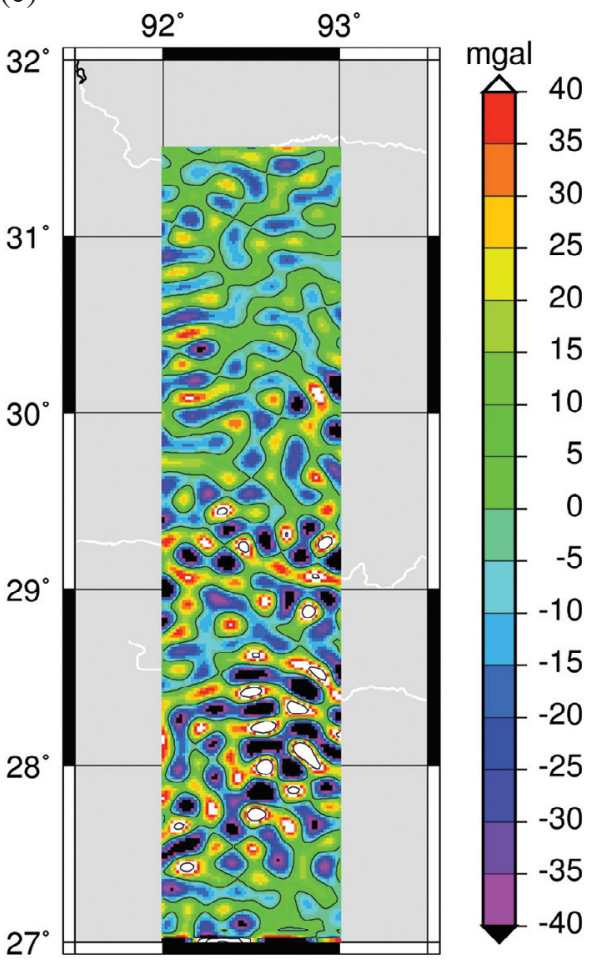

Fig. 7. Differences between downward continued and "true" gravity values (from EGM08, see text) at different altitudes in Tibet, from (a) Case G, (b) Case H, and (c) Case I.

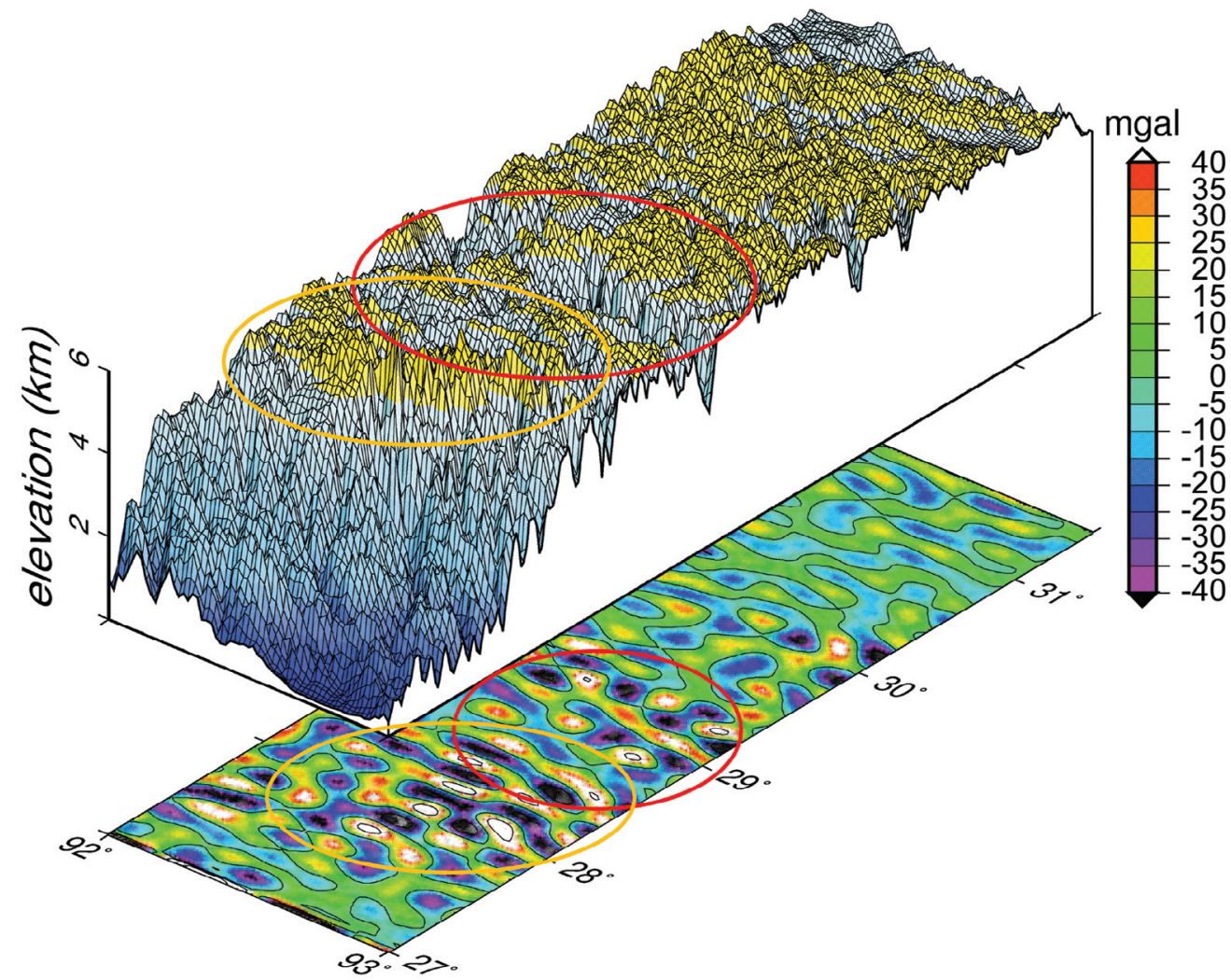

Fig. 8. Differences between downward-continued gravity and ground gravity (lower part) and the topography. 

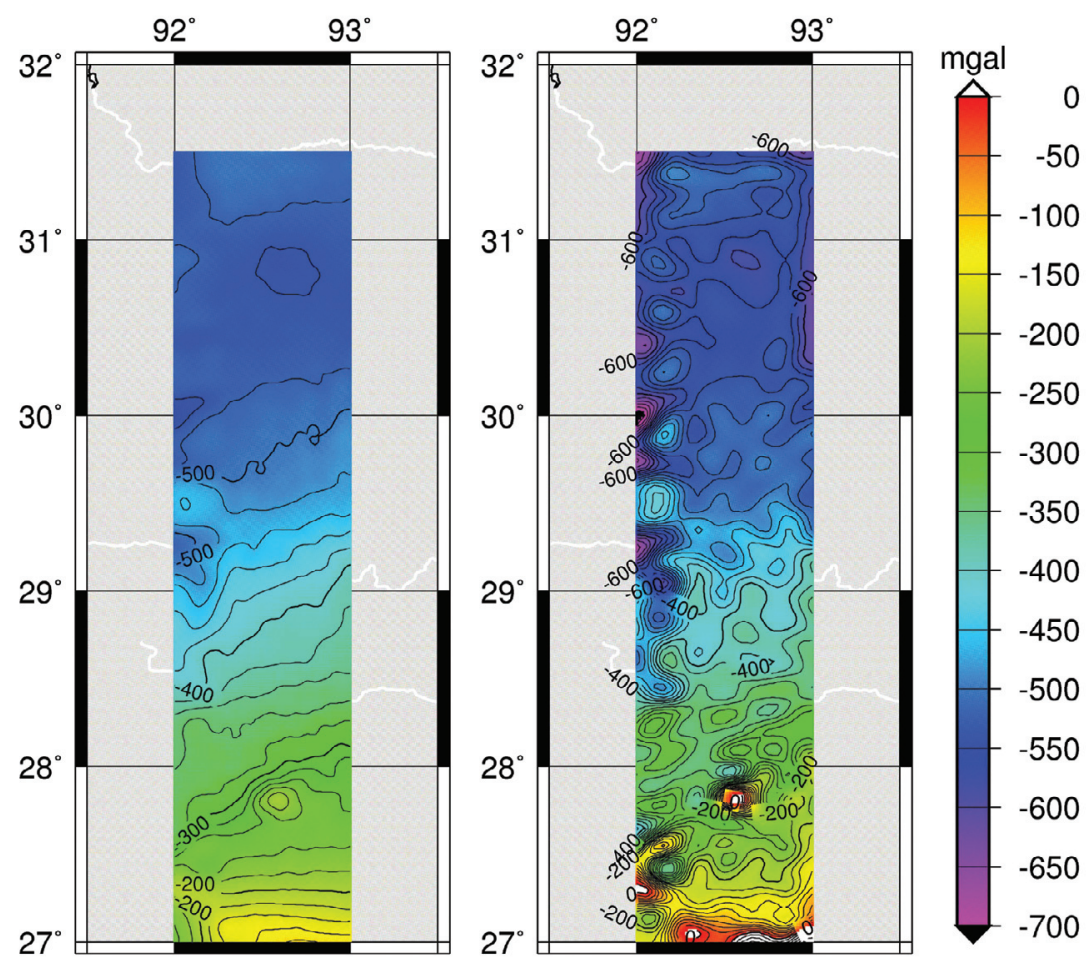

Fig. 9. Bouguer anomalies of Tibet at $10 \mathrm{~km}$ (left) and downward continued values at sea level.

clear. To see the enhancement and the downward continuation limitation, we computed the BA in the following cases along a profile over a rapid-varying terrain:

Case 1: BA at $10 \mathrm{~km}$;

Case 2a: BA at sea level, downward continued from BA of Case $1, \alpha=15$;

Case 2b: same as Case 2a, but $\alpha=20$;

Case 3: BA at sea level, computed directly from the spherical harmonic expansion of EGM08 to degree 2160 at sea level.

Figure 10 shows the along-profile BA in all cases. In general, the BA along this profile decreases with increasing latitude and this trend is consistent with the result given by Jin et al. (1994). Compared to Case 3, the BA in Case 1 significantly lost gravity amplitude, especially near $27.5^{\circ}$ north. In general, the gravity variation weakens towards the north. Again, the downward continuation enhances the high-frequency signature (Case 1 vs. Case 2a), but the extent of enhancement is affected by parameter $\alpha$ : the degree of smoothness increases with the value of $\alpha$ (Case $2 b$ vs. Case 2a). Despite the flight altitude attenuation effect, the long wavelength features are preserved in the gravity field obtained at $10 \mathrm{~km}$. This feature preservation is evidenced by the similar, large-scaled trends of BA in Cases 1 and 3.

Figure 11 shows the power spectra of along-profile BA in Cases 1, 2a, and 3. Except at wavelengths around $10 \mathrm{~km}$, the spectra patterns from Cases 1 and 3 are similar, but Case 1 has lower magnitudes. Downward continuation increases the powers at $10 \mathrm{~km}$ (Case 1) to powers at sea level (Case 2a). At the low-frequency band (wavelengths $>19 \mathrm{~km}$ ) and the high-frequency band (wavelengths $<7 \mathrm{~km}$ ), the powers of Case $2 \mathrm{a}$ agree better with the powers of Case 3, in comparison to Case 1. This suggests that downward continuation can partially restore the gravity signals at these two bands from the gravity data at $10 \mathrm{~km}$. In many cases, the sought-for tectonic features are at wavelengths longer than $100 \mathrm{~km}$ (Jin et al. 1994), which is far larger than the resolvable wavelength of gravity from an airborne gravity survey at $10 \mathrm{~km}$. Therefore, an airborne gravity survey in Tibet will no doubt deliver gravity data to advance the understanding of Tibetan tectonic structures. However, for a potential airborne gravity survey in Tibet, we recommend that we should always use the free-air gravity or Bouguer gravity data at the flight altitude when such data are to be used for geophysical analyses. This is to avoid the error introduced by downward continuation as seen in Table 5 . For example, Hwang et al. (2007) has demonstrated a rigorous method to compute Bouguer gravity anomalies at any altitude.

\section{CONCLUSIONS}

This paper used in situ airborne gravity data collected at three altitudes in Alabama to investigate gravity accura- 
cies under different upward and downward continuation scenarios. The 3D Fourier series of local gravity field representation results in continuation errors at few mgal level in Alabama, even in the extreme case of downward continuation from $11 \mathrm{~km}$ to sea level. Similar investigations were made in Tibet using EGM08-derived airborne gravity data at
0,5 , and $10 \mathrm{~km}$. Tibet has a much more rugged terrain than Alabama, making the downward/upward continuation outcomes in these two regions different. However, our investigation results (see Figs. 10 and 11 and the corresponding arguments in the later part of section 4) strongly support the need for an airborne gravity survey in Tibet. In summary,

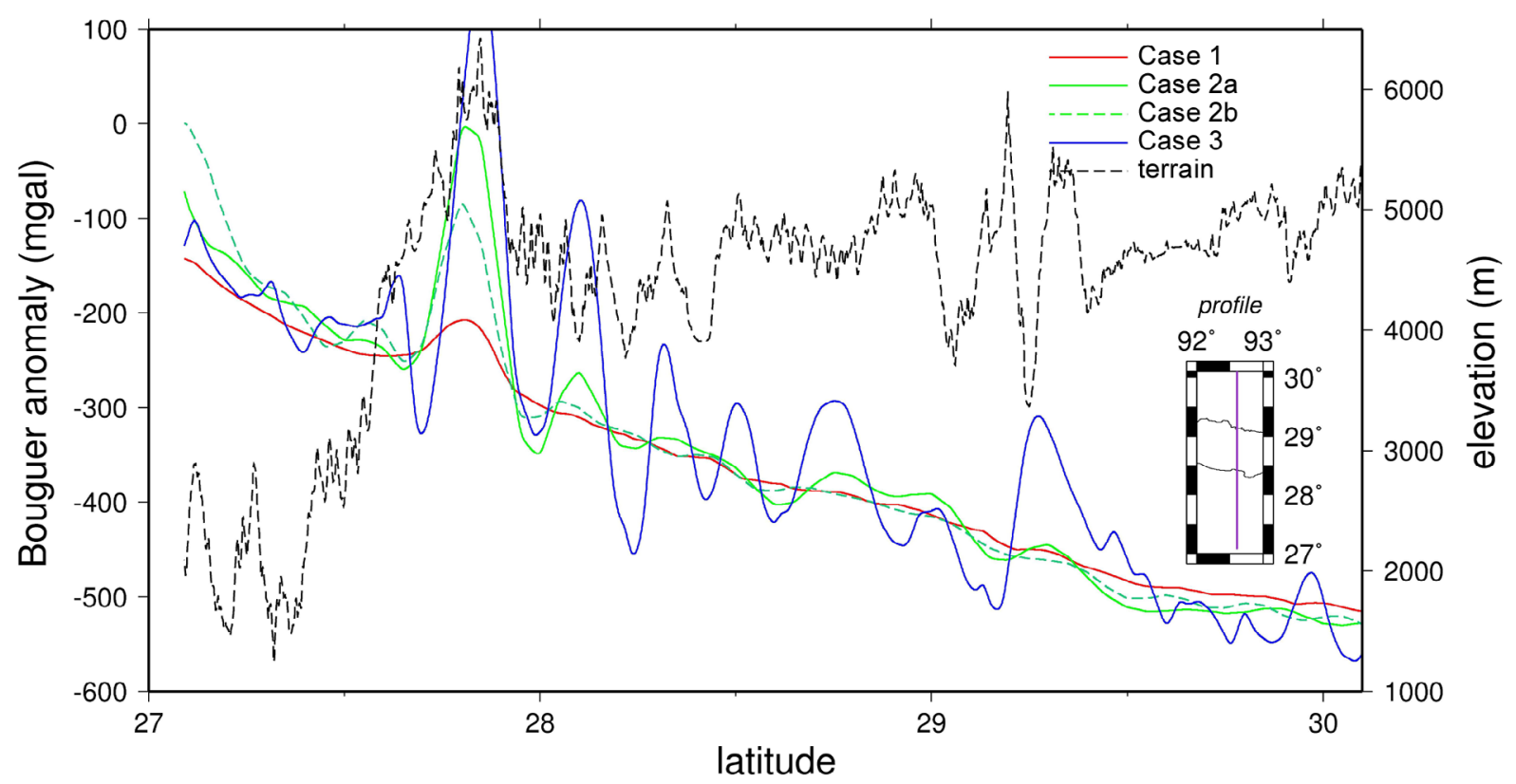

Fig. 10. Along-profile Bouguer anomalies in four cases (see text). The inserted map shows the profile location.

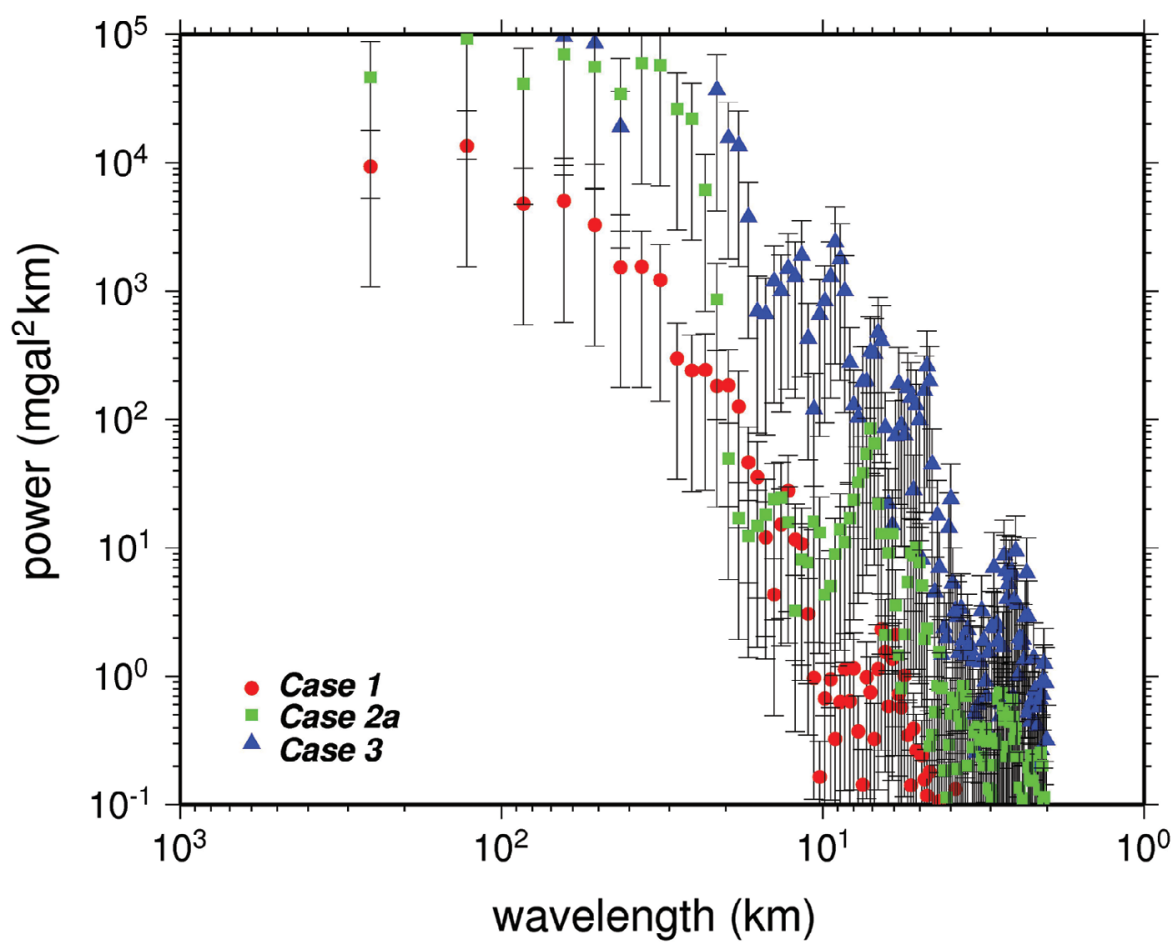

Fig. 11. Power spectra of along-profile Bouguer anomalies in three cases (see text). 
the value of such a survey is supported by the following facts:

(1) Collection of gravity values directly on the surface of Tibet may be possible only in areas with manageable transportation, making the gravity data distribution uneven.

(2) With a flight altitude of $10 \mathrm{~km}$ in Tibet, an airborne gravity survey can deliver a gravity field over the entire Tibet region with uniform spatial resolution and gravity accuracy (about $1-2$ mgal at the flight altitude). In addition, a $10-\mathrm{km}$ altitude survey over Tibet is of a high enough altitude to avoid most of the orographic turbulence that an aircraft would experience when flying within 1000 $2000 \mathrm{~m}$ of the topography. For example, GRAV-D experienced much turbulence when flying at altitudes close to the Alaska Range Mountains in 2009 and 2010.

(3) Certain local features in the Tibetan airborne gravity field can be enhanced by downward continuation field (see Fig. 10).

Finally, because of the relatively large errors caused by downward continuation in such a rugged terrain as Tibet, we suggest that, whenever possible, the free-air or Bouguer gravity values at the flight altitude, rather than downward continued values, be used for geophysical and geodetic applications.

Acknowledgements This study is supported by National Land Surveying and Mapping Center, Ministry of the Interior, Taiwan, under the project "Nearshore shipborne gravity survey," and by NSFC (mainland China) under grant No. 41128003. The support from the National Geodetic Survey, USA, is administrated under the GRAV-D project. We thank three anonymous referees for their reviews that improved the quality of this paper.

\section{REFERENCES}

Braitenberg, C., M. Zadro, J. Fang, Y. Wang, and H. T. Hsu, 2000: The gravity and isostatic Moho undulations in Qinghai-Tibet plateau. J. Geodyn., 30, 489-505, doi: 10.1016/S0264-3707(00)00004-1. [Link]

Buttkus, B., 2000: Spectral Analysis and Filter Theory in Applied Geophysics, Springer, Berlin, 668 pp.

GRAV-D Science Team, 2011a: Gravity for the Redefinition of the American Vertical Datum (GRAV-D) Project, Airborne Gravity Data. Block CS01, available at http://www.ngs.noaa.gov/GRAV-D/data cs01.shtml.

GRAV-D Science Team, 2011 b: Block CS01 (Central South 01); GRAV-D Airborne Gravity Data User Manual. Theresa Diehl (Ed.), version 1, available at http://www. ngs.noaa.gov/GRAV-D/data cs01.shtml.

Heiskanen, W. A. and H. Moritz, 1967: Physical Geodesy, W. H. Freeman \& Co Ltd, San Francisco and London, $364 \mathrm{pp}$.
Hwang, C., Y. S. Hsiao, H. C. Shih, M. Yang, K. H. Chen, R. Forsberg, and A. V. Olesen, 2007: Geodetic and geophysical results from a Taiwan airborne gravity survey: Data reduction and accuracy assessment. J. Geophys. Res., 112, B04407, doi: 10.1029/2005JB004220. [Link]

Hwang, C., H. C. Shih, Y. S. Hsiao, and C. H. Huang, 2012: Airborne gravity surveys over Taiwan Island and Strait, Kuroshio Current and South China Sea: Comparison of GPS and gravity accuracies at different flight altitudes. Mar. Geodesy, 35, 287-305, doi: 10. 1080/01490419.2011.634962. [Link]

Jin, Y., M. K. McNutt, and Y. Zhu, 1994: Evidence from gravity and topography data for folding of Tibet. $\mathrm{Na}$ ture, 371, 669-674, doi: 10.1038/371669a0. [Link]

Jordan, T. A., F. Ferraccioli, D. G. Vaughan, J. W. Holt, H. Corr, D. D. Blankenship and T. M. Diehl, 2010: Aerogravity evidence for major crustal thinning under the Pine Island Glacier region (West Antarctica). Geol. Soc. Am. Bull., 122, 714-726, doi: 10.1130/B26417.1. [Link]

Koch, K.-R., 1999: Parameter Estimation and Hypothesis Testing in Linear Models, Springer, Berlin, 333 pp.

Liebelt, P. B., 1967: An Introduction to Optimal Estimation, Addison-Wesley Pub. Co, 273 pp.

Olesen, A. V., R. Forsberg, K. Keller, and A. Gidskehaug, 2000: Airborne gravity survey of Lincoln Sea and Wandel Sea, North Greenland. Phys. Chem. Earth, 25, 25-29, doi: 10.1016/S1464-1895(00)00005-3. [Link]

Pail, R., S. Bruinsma, F. Migliaccio, C. Förste, H. Goiginger, W.-D. Schuh, E. Höck, M. Reguzzoni, J. M. Brockmann, O. Abrikosov, M. Veicherts, T. Fecher, R. Mayrhofer, I. Krasbutter, F. Sansò, and C. C. Tscherning, 2011: First GOCE gravity field models derived by three different approaches. J. Geodesy, 85, 819-843, doi: 10.1007/s00190-011-0467-x. [Link]

Pavlis, N. K., S. A. Holmes, S. C. Kenyon, and J. K. Factor, 2012: The development and evaluation of the Earth Gravitational Model 2008 (EGM2008). J. Geophys. Res., 117, B04406, doi: 10.1029/2011JB008916. [Link]

Shen, W. B., J. Li, B. Tian, and J. Han, 2011: Determination of the quasi-geoid of Xinjiang and Tibet areas and the normal height of Mt. Everest based on EGM2008. Terr. Atmos. Ocean. Sci., 22, 121-131, doi: 10.3319/ TAO.2010.07.26.01(TibXS). [Link]

Shin, Y. H., H. Xu, C. Braitenberg, J. Fang, and Y. Wang, 2007: Moho undulations beneath Tibet from GRACEintegrated gravity data. Geophys. J. Int., 170, 971-985, doi: 10.1111/j.1365-246X.2007.03457.x. [Link]

Tseng, T. L., W. P. Chen, and R. L. Nowack, 2009: Northward thinning of Tibetan crust revealed by virtual seismic profiles. Geophys. Res. Lett., 36, L24304, doi: 10.1029/2009GL040457. [Link]

Verdun, J., E. E. Klingelé, R. Bayer, M. Cocard, A. Geiger, 
and H.-G. Kahle, 2003: The alpine Swiss-French airborne gravity survey. Geophys. J. Int., 152, 8-19, doi: 10.1046/j.1365-246X.2003.01748.x. [Link]

Wang, Y. M., D. R. Roman, and J. Saleh, 2008: Analytical downward and upward continuation based on the method of domain decomposition and local functions. Int. Assoc. Geodesy Symp., 132, 356-360, doi: 10.1007/97 8-3-540-74584-6_58. [Link] 